\title{
MONOTONE CLONES, RESIDUAL SMALLNESS AND CONGRUENCE DISTRIBUTIVITY
}

\author{
RALPH MCKENZIE
}

\begin{abstract}
Corresponding to each ordered set there is a variety, determined up to equivalence, generated by an algebra whose term operations are all the monotone operations on the ordered set. We produce several characterisations of the finite bounded ordered sets for which the corresponding variety is congruence-distributive. In particular, we find that congruence-distributivity, congruence-modularity, and residual smallness are equivalent for these varieties.
\end{abstract}

\section{INTRODUCTION}

We say that an algebra $\mathbf{A}=\left\langle P, f_{i}(i \in I)\right\rangle$ is monotone with respect to an ordered set $\mathbf{P}=\langle P, \leqslant\rangle$ if every fundamental operation $f_{i}$ of $\mathbf{A}$ preserves the order of $\mathbf{P}$. An algebra $\mathbf{A}$ is order-primal with respect to $\mathbf{P}$ if $\mathbf{A}$ is monotone with respect to $\mathbf{P}$ and the clone $M(\mathbf{P})$, consisting of all operations monotone with respect to $\mathbf{P}$, is identical with the clone of term operations of $\mathbf{A}$. An algebra order-primal with respect to $\mathbf{P}$ will be denoted by $\mathbf{A}(\mathbf{P})$. We say that $\mathbf{P}$ is bounded if it has a least and a largest element. The variety $\boldsymbol{V}(\mathbf{A}(\mathbf{P}))$ generated by an order-primal algebra for a (bounded) ordered set $\mathbf{P}$ will be called a (bounded) order-primal variety.

Martynjuk [15] proved that the order-primal algebra corresponding to a finite bounded ordered set $\mathbf{P}$ is pre-primal; that is, $\mathrm{M}(\mathbf{P})$ together with any operation over $P$ that lies outside of $M(P)$ generates the clone of all operations on $P$. Of the six classes of pre-primal algebras delineated in Rosenberg [20], those associated with bounded ordered sets are the only ones for which answers to straightforward questions about the algebra, its clone, and the variety it generates, are often very difficult to answer. For general studies of the varieties generated by pre-primal algebras, consult Denecke [7], Knoebel [13] and Lau [14].

Our motive for writing this paper was to prove that the SI conjecture holds for the bounded order-primal varieties. The SI conjecture is the statement that a variety $\mathcal{V}$

Received 27 April 1989.

Research supported by National Science Foundation grant DMS 8302255.

Copyright Clearance Centre, Inc. Serial-fee code: 0004-9729/90 \$A2.00+0.00. 
generated by a finite algebra either possesses no subdirectly irreducible algebras of more than $n-1$ elements, for some integer $n$ (that is, $\mathcal{V}$ is residually $<n$ ), or $\mathcal{V}$ possesses a subdirectly irreducible algebra of cardinality greater than $\lambda$ for every cardinal $\lambda$ (that is, $\mathcal{V}$ is residually large). For pre-primal algebras other than the order-primal algebras, it has been known for some time that the clone of term operations is finitely generated, and that the variety generated by the algebra is residually bounded by some integer (see [13]). In this paper, we prove that the SI conjecture does hold for bounded order-primal varieties, and in fact holds for varieties generated by finite monotone algebras belonging to more extensive classes. For these varieties, residual smallness is equivalent to congruence-distributivity. (See Theorem 1.1, Corollary 1.2 and Theorem 2.4.) By well-known results of Jónsson [12], if $\boldsymbol{V}(\mathbf{A})$ is congruence-distributive then every subdirectly irreducible algebra in this variety is a homomorphic image of some subalgebra of $\mathbf{A}$. The SI conjecture has by now been proved for some diverse and very broad families of algebras (see [11, Chapter 10]); our expectation is that it is true for all finite algebras.

The study of monotone operations on ordered sets offers some fascinating problems for the clone-theorist or universal algebraist. Besides our main result, this paper contains several minor contributions to the study. We find in Section 2 some equivalent conditions for $\boldsymbol{V}(\mathbf{A}(\mathbf{P}))$ to be congruence-distributive. In Section 3 we survey briefly some of the literature and make some comments on what appear to be the two outstanding current open problems in this area, namely, for which $P$ is the clone $M(P)$ finitely generated? and under what conditions does $\boldsymbol{V}(\mathbf{A}(\mathbf{P})$ ) satisfy some interesting Maltsev condition such as congruence-distributivity, or possession of a near-unanimity operation?

\section{WhEn is $\boldsymbol{V}(\mathbf{A}(\mathbf{P}))$ RESidually SMall?}

We prove in this section the following theorem. We are most interested in a corollary which we shall state and prove immediately. Following a brief digression, we will prove the theorem.

Theorem 1.1. Suppose that $\mathbf{P}$ is a bounded ordered set and $\mathbf{A}$ is an algebra monotone with respect to $\mathbf{P}$ that has term operations $D(x, y, z)$ and $U(x, y, z)$ such that these equations are valid in $\mathrm{A}$, where 0 and 1 are the least and largest elements of $\mathbf{P}$ :

$$
\begin{aligned}
& D(0,0, x) \approx 0, D(0, x, x) \approx D(x, 0, x) \approx D(x, x, x) \approx x \quad \text { and } \\
& U(1,1, x) \approx 1, U(1, x, x) \approx U(x, 1, x) \approx U(x, x, x) \approx x .
\end{aligned}
$$

Then, if $\boldsymbol{V}(\mathbf{A})$ is residually small, it is congruence-distributive. 
Corollary 1.2. If $\mathbf{P}$ is a finite bounded ordered set and $\mathcal{V}=\boldsymbol{V}(\mathbf{A}(\mathbf{P})$ ) where $\mathbf{A}(\mathbf{P})$ is order-primal with respect to $\mathbf{P}$, then $\mathcal{V}$ is residually small if and only if it is congruence-distributive.

Proof: We can easily define monotone operations on $\mathbf{P}$ that satisfy (1) and (2). For example, let $D(x, y, z)=z$ unless $x=y=0$, and define $D(0,0, z)=0$. Define $U(x, y, z)$ dually. The operations $D$ and $U$ are term operations of $\mathbf{A}(\mathbf{P})$. Hence Theorem 1.1 supplies the fact that residual smallness implies congruence-distributivity. As we remarked earlier, if $\mathcal{V}$ is congruence-distributive then it is residually $<|A|+1$. $\square$

Corollary 1.2 will be strengthened in Theorem 2.4 where the hypotheses of boundedness will be weakened by a half.

Before we begin our proof of Theorem 1.1, we discuss briefly a related result that was proved by Davey, Quackenbush and Schweigert in [3]. Their result had to do with finite ordered sets $\mathbf{P}$ that may not be bounded. Note that $\mathbf{A}(\mathbf{P})$ has no proper subalgebras and has, up to isomorphism, at most three homomorphic images. There is a congruence $\theta$ corresponding to the partition of $\mathbf{P}$ into its connected components; we write $\mathbf{A}^{\prime}(\mathbf{P})$ for $\mathbf{A}(\mathbf{P}) / \theta$. If $\mathbf{P}$ is connected or is discrete (that is, is an antichain), then $\mathbf{A}(\mathbf{P})$ is a simple algebra $\left(\theta=1_{P}\right.$ or $0_{P}$ respectively). Otherwise, $\theta$ is the unique non-trivial congruence, $\mathbf{A}(\mathbf{P})$ is subdirectly irreducible, and $\mathbf{A}^{\prime}(\mathbf{P})$ is simple. The result of the three authors was that $\mathbf{V}(\mathbf{A}(\mathbf{P}))$ is congruence-distributive provided that every subdirectly irreducible algebra in this variety is isomorphic either to $\mathbf{A}(\mathbf{P})$ or to $\mathbf{A}^{\prime}(\mathbf{P})$, in other words, provided that $\boldsymbol{V}(\mathbf{A}(\mathbf{P}))=\boldsymbol{S P}\left\{\mathbf{A}(\mathbf{P}), \mathbf{A}^{\prime}(\mathbf{P})\right\}$. To prove it, they invoked generalised duality theory in a rather long argument to prove that the quasi-variety $\boldsymbol{S P}\left\{\mathbf{A}(\mathbf{P}), \mathbf{A}^{\prime}(\mathbf{P})\right\}$ is relatively congruence-distributive. We now digress briefly from our main goal in order to prove a slightly more general result by a substantially different argument.

ThEOREM 1.3. Let $\left\{\mathbf{A}_{1}, \ldots, \mathbf{A}_{k}\right\}$ be a finite collection of finite order-primal algebras, all of the same similarity type. The quasi-variety $\mathcal{K}=\boldsymbol{S P}\left\{\mathbf{A}_{1}, \ldots, \mathbf{A}_{k}\right\}$ is relatively congruence-distributive.

PROOF: Our proof is actually an easy application of the tame congruence theory of Hobby and McKenzie [11]. To prove that $\mathcal{K}$ is relatively congruence-distributive, it suffices to prove that for finite $B \in \mathcal{K}$, the lattice $C_{\mathcal{K}} \mathbf{B}$ is distributive. $\mathbf{C o n}_{\mathcal{K}} \mathbf{B}$ consists of the congruences $\theta \in$ Con $B$ such that $B / \theta \in \mathcal{K}$, ordered by inclusion. Let $C$ denote the set of all congruences $\delta$ of $\mathbf{B}$ such that $\mathrm{B} / \delta \cong \mathbf{A}_{i}$ for some $i \leqslant k$. Then the members of $C_{\mathcal{X}} B$ are precisely the congruences of $B$ that can be expressed as the intersection of some subset of $C$ (since each $\mathbf{A}_{i}$ has no proper subalgebras). From this fact, it is easy to see that the distributivity of $\operatorname{Con}_{\mathcal{K}} B$ will follow if we can show that each $\delta \in C$ is a meet-prime element of Con B. Assume that $B$ is finite, $\delta \in$ Con B 
and $\mathbf{B} / \delta \cong \mathbf{A}_{i}$. We can obviously further assume that $\mathbf{A}_{i}$ has at least two elements. We remarked earlier that an order-primal algebra has at most three congruences. Thus the algebra $\mathbf{A}_{i}$ is subdirectly irreducible. We claim that its monolith $\beta$ is a type 3 or 4 cover of $0_{A_{i}}$, and that the $\left\langle 0_{A_{i}}, \beta\right\rangle$-minimal sets in $\mathbf{A}_{i}$ have no tails. What this means is just that there are two distinct $\beta$-equivalent elements $c, d \in A_{i}$ and a polynomial operation $p(x)$ of $\mathbf{A}_{i}$ such that $p\left(A_{i}\right)=\{c, d\}$ and $p(p(x))=x$ ( $p$ is idempotent), and there are polynomial operations $j(x, y)$ and $m(x, y)$ such that, restricted to $\{c, d\}$, these polynomials become the join and meet of a two-element lattice. Since the construction of polynomials of $\mathbf{A}_{i}$ amounts to finding functions that are monotone with respect to the underlying order, our claim is easily established. Now the argument used to prove Lemma 14.4 in [11], with very slight and obvious modifications, will prove that whenever $\gamma, \lambda \in$ Con B and $\gamma \cap \lambda \leqslant \delta$, then either $\gamma \leqslant \delta$ or $\lambda \leqslant \delta$. This ends the proof.

Before launching into the proof of Theorem 1.1, we recall the standard Maltsev condition for congruence-distributivity (see Jónsson [12]). A variety $\mathcal{V}$ is congruencedistributive if and only if for some positive integer $n$ there are terms $d_{0}(x, y, z), \ldots$, $d_{n}(x, y, z)$ in the language of $\mathcal{V}$ (called Jónsson terms for $\mathcal{V}$ ) such that these equations (the Jònsson equations) are valid in $\mathcal{V}$

$$
\begin{gathered}
x \approx d_{0}(x, y, z) \approx d_{i}(x, y, x) \approx d_{n}(z, y, x)(\text { for } 0 \leqslant i \leqslant n) \\
d_{2 i}(x, x, y) \approx d_{2 i+1}(x, x, y)(\text { for } 0 \leqslant i \leqslant(n-1) / 2) \\
d_{2 i-1}(x, y, y) \approx d_{2 i}(x, y, y)(\text { for } 1 \leqslant i \leqslant n / 2)
\end{gathered}
$$

Thus an order-primal algebra $\mathbf{A}(\mathbf{P})$ generates a congruence distributive variety if and only if $\mathbf{P}$ admits some system of monotone $\mathbf{3}$-ary operations that obey Jónsson's equations.

LemmA 1.4. Let $\mathbf{A}$ be a monotone algebra with respect to an ordered set $\mathbf{P}$ that has a zero element 0 . Suppose that $\mathbf{A}$ possesses a term operation $D(x, y, z)$ satisfying equations (1) from Theorem 1.1. If $\mathbf{V}(\mathbf{A})$ is residually small then $\mathbf{A}$ possesses term operations $d_{0}(x, y, z), \ldots, d_{n}(x, y, z)$ satisfying $d_{i}(x, 0, x) \approx x$ (for all $i \leqslant n$ ) and satisfying all of Jónsson's equations $(3)$ except $d_{i}(x, y, x) \approx x$.

Proof: Choose an infinite cardinal $\lambda$ that is larger than the cardinality of any subdirectly irreducible algebra in $\mathcal{V}=\boldsymbol{V}(\mathbf{A})$. We shall construct an algebra in $\mathcal{V}$ that is a likely candidate to be a subdirectly irreducible algebra of cardinality $\lambda$. Then we shall show that the failure of our construction actually to produce a subdirectly irreducible algebra of cardinality $\lambda$ yields the existence of the desired term operations.

To begin, we set

$$
X=P \times P \times \lambda
$$


where $P$ is the universe of $\mathbf{A}$. We define functions $f_{\ell}, f_{r}, f_{\ell, u}, f_{r, u} \in P^{X}(u \in \lambda)$. For $x=(a, b, v)$ in $X$ we define $f_{\ell}(x)=a$ and $f_{r}(x)=b$; if $u \neq v$ we define $f_{\ell, u}(x)=a$ and $f_{r, u}(x)=b$, while if $u=v$ we put $f_{l, u}(x)=f_{r, u}(x)=0$. We define $Q$ to be the subalgebra of $\mathbf{A}^{x}$ generated by the set

$$
\left\{f_{\ell}, f_{r}\right\} \cup\left\{f_{\ell, u}, f_{r, u}: u \in \lambda\right\}
$$

and define $\theta$ to be the congruence of $Q$ generated by the set

$$
\left\{\left(f_{\ell, u}, f_{r, u}\right): u \in \lambda\right\}
$$

of ordered pairs of elements of $\mathbf{Q}$.

We now show that since $\mathcal{V}$ is residually $<\lambda$, then $\left(f_{\ell}, f_{r}\right) \in \theta$. Suppose that this fails. Then choose $\tau$ to be any congruence of $Q$ that is maximal in the set of all congruences containing $\theta$ that do not contain the pair $\left(f_{\ell}, f_{r}\right)$. Thus the algebra $\mathbf{S}=\mathbf{Q} / \tau$ is certainly subdirectly irreducible. We shall see that $|\mathbf{S}| \geqslant \lambda$. If it were the case that $|\mathbf{S}|<\lambda$, then there would exist some $u, v \in \lambda, u \neq v$, such that $f_{\ell, u} \equiv f_{\ell, v}$ $(\bmod \tau)$; and consequently, $f_{r, u} \equiv f_{r, v}(\bmod \tau)$. Let $D$ denote the term operation of $\mathbf{Q}$ induced by the term operation $D(x, y, z)$ of $\mathbf{A}$. From equations (1) satisfied by $D(x, y, z)$ in $\mathbf{A}$, it follows that for $\varepsilon \in\{\ell, r\}$, in $\mathbf{Q}$ we have

$$
\begin{aligned}
& D\left(f_{e, u}, f_{\varepsilon, u}, f_{\varepsilon}\right)=f_{e, u} \quad \text { and } \\
& D\left(f_{e, u}, f_{\varepsilon, v}, f_{\varepsilon}\right)=f_{e} .
\end{aligned}
$$

Then since $f_{e, u} \equiv f_{e, v}(\bmod \tau)$, we have $f_{e} \equiv f_{e, u}(\bmod \tau)$. Since $f_{\ell, u} \equiv f_{r, u}(\bmod \theta)$, then we can infer that $f_{\ell} \equiv f_{r}(\bmod \tau)$. But this contradicts our choice of $\tau$ to not contain the pair $\left(f_{\ell}, f_{r}\right)$. We conclude that $\left(f_{\ell}, f_{r}\right) \in \theta$.

Using the fact just proved, we now proceed to deduce the existence of the desired three-variable term operations. Since $\left(f_{\ell}, f_{r}\right) \in \theta$, then there exists a finite sequence

$$
g_{0}=f_{\ell}, g_{1}, \ldots, g_{m}=f_{r}
$$

of elements of $\mathrm{Q}$, and for each $s<m$ a binary polynomial operation $F_{s}(x, y)$ of $\mathrm{Q}$, and some $i, \in \lambda$ such that

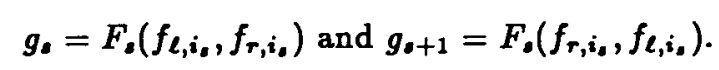

Notice that for each of the generators $f$ of $\mathbf{Q}$ there is a term operation $B(x, y)$ of $\mathbf{A}$ such that for all $x=(a, b, u) \in X$ we have $f(x)=B(a, b)$ unless $f=f_{\ell, u}$ or $f=f_{r, u}$. (We can take either $B(x, y)=x$ or $B(x, y)=y$.) Consequently, for every $f$ in $Q$ there is a term operation $B(x, y)$ and a finite set $L \subseteq \lambda$ such that

$$
B(a, b)=f(x) \text { for all } x=(a, b, u) \in X \text { such that } u \notin L .
$$


For $s<m$, we shall denote the operation $B(x, y)$ corresponding in this way to $g$, by $B_{a}(x, y)$. Thus we have $B_{0}(x, y)=x$ and $B_{m}(x, y)=y$, and

$$
B_{\lrcorner}(a, b)=F_{d}\left(f_{\ell, i_{d}}(x), f_{r, i_{d}}(x)\right) \text { and } B_{o+1}(a, b)=F_{s+1}\left(f_{r, i_{d}}(x), f_{\ell, i_{d}}(x)\right)
$$

for all $x=(a, b, u) \in X$ such that $u \notin L$, where $L$, is a certain finite subset of $\lambda$.

Choose any $s<m$ and hold it fixed for a time. Notice that the polynomial operation $F_{a}(x, y)$ of $(4)$ and (5) can be expressed as

$$
F_{\bullet}(z, w)=K_{\bullet}\left(z, w, f_{\ell, i_{\ell}}, f_{r, i_{\ell}}, f_{\ell, j_{0}}, f_{r, j_{0}}, \ldots, f_{\ell, j_{k-1}}, f_{r, j_{k-1}}, f_{\ell}, f_{r}\right)
$$

for some $k$, some $2 k+6$-ary term operation $K$ of $\mathbf{A}$, and some distinct elements $j_{0}, \ldots, j_{k-1} \in \lambda$ that are all distinct from $i_{\text {. }}$. Evaluating the equations (4), (5), (6) at any $x \in X$ of the form $x=(a, b, u)$ where $u \notin L, \cup\left\{i_{\varepsilon}, j_{0}, \ldots, j_{k-1}\right\}$, we find that

$$
\begin{aligned}
B_{s}(a, b) & =K_{s}(a, b, a, b, \ldots, a, b) \quad \text { and } \\
B_{\bullet+1}(a, b) & =K_{s}(b, a, a, b, \ldots, a, b) .
\end{aligned}
$$

Defining $L_{s}(x, y, u, v)=K,(x, y, u, v, u, v, \ldots, u, v)$, this becomes

$$
B_{\triangleleft}(a, b)=L_{\diamond}(a, b, a, b), B_{\triangleleft+1}(a, b)=L_{\triangleleft}(b, a, a, b) .
$$

Our next task is to show that the 4 -ary operation $L$, of $\mathbf{A}$ obeys the equations

$$
L_{\diamond}(0,0, z, z)=L_{\triangleleft}(0, z, z, z)=L_{\triangleleft}(z, 0, z, z)=L_{\triangleleft}(z, z, z, z)=z .
$$

First notice that every pair of generators of $\theta$ agree at any $x \in X$ of the form $(a, a, u)$, and therefore

$$
g_{s}(x)=g_{s+1}(x)=g_{0}(x)=a, \quad \text { for } x=(a, a, u) \in X .
$$

Then evaluating all the functions in equations (4) and (6) at $x=(a, a, i$,$) and at$ $x=(a, a, u)$ for some $u \notin\left\{i_{e}, j_{0}, \ldots, j_{k-1}\right\}$, we find that

$$
K_{\diamond}(0,0,0,0, a, a, \ldots, a, a)=a=K_{,}(a, a, a, a, \ldots, a, a) .
$$

The equations (9) clearly follow from this and from our definition of $L_{\mathbb{A}}$, since $K_{\mathbb{a}}$ is monotone.

Now we define

$$
D_{2 \diamond+1}(x, y, z)=L_{\triangleleft}(y, z, x, z), D_{2 s+2}(x, y, z)=L_{\triangleleft}(z, y, x, z) .
$$


Since we have been considering an arbitrary $s<m$, we can now regard $D_{i}(x, y, z)$ as having been defined via $(10)$ for $1 \leqslant i \leqslant 2 m$. It is easy to check that the equations ( 8 ) and (10) imply

$$
\begin{aligned}
D_{2 s+1}(x, z, z) & =D_{2 \triangleleft+2}(x, z, z) & & \text { for } 0 \leqslant s<m, \text { and } \\
D_{2 \triangleleft}(x, x, z) & =B_{s}(x, z)=D_{2 \vartheta+1}(x, x, z) & & \text { for } 1 \leqslant s<m .
\end{aligned}
$$

The equations (9) and (10) imply that $D_{i}(x, 0, x)=x$ for all $1 \leqslant i \leqslant 2 m$. Moreover, $D_{1}(x, x, z)=B_{0}(x, z)=x$ and $D_{2 m}(x, x, z)=B_{m}(x, z)=z$. Therefore, taking $D_{0}(x, y, z)=x$ and $D_{2 m+1}(x, y, z)=z$, the operations $D_{0}, D_{2}, \ldots, D_{2 m+1}$ satisfy all the required equations. This finishes our proof of the lemma.

Proof of Theorem 1.1: We assume that $\mathbf{P}$ and $\mathbf{A}$ satisfy all the hypotheses of Theorem 1.1, and that $\boldsymbol{V}(\mathbf{A})$ is residually small. According to Lemma 1.4, there are term operations $D_{0}(x, y, z), \ldots, D_{n}(x, y, z)$ of $\mathbf{A}$ satisfying all of Jónsson's equations except $D_{i}(x, y, x)=x$, and satisfying $D_{i}(x, 0, x)=x$. The dual of Lemma 1.4 is also valid, and since our hypotheses are self-dual, it yields the existence of another system of term operations $U_{0}(x, y, z), \ldots, U_{m}(x, y, z)$ satisfying all of Jónsson's equations except $U_{i}(x, y, x)=x$, and satisfying $U_{i}(x, 1, x)=x$. We define

$$
F_{i, j}(x, y, z)=D_{i}\left(x, U_{j}(x, y, z), z\right) .
$$

Notice that $U_{j}(a, 1, a)=a$ implies $U_{j}(a, b, a) \leqslant a$; then since $D_{i}(a, 0, a)=D_{i}(a, a, a)$ $=a$ and $D_{i}$ is monotone, we have that $F_{i, j}(a, b, a)=D_{i}\left(a, U_{j}(a, b, a), a\right)=a$. Thus the $F_{i, j}(x, y, z)$ satisfy the crucial Jónsson equation that we were unable to ensure the $D_{i}(x, y, z)$ and $U_{j}(x, y, z)$ would satisfy.

We also have

$$
\begin{aligned}
F_{1,0}(x, y, z)= & D_{1}(x, x, z)=x, F_{k, 2 j}(x, x, z)=F_{k, 2 j+1}(x, x, z), \\
& F_{k, 2 j+1}(x, z, z)=F_{k, 2 j+2}(x, z, z), \\
F_{2 i+1, m}(x, y, z)= & D_{2 i+1}(x, z, z)=D_{2 i+2}(x, z, z)=F_{2 i+2, m}(x, y, z), \\
& F_{2 i, 0}(x, y, z)=F_{2 i+1,0}(x, y, z) .
\end{aligned}
$$

and

Thus the sequence

$$
\begin{gathered}
F_{1,0}, \ldots, F_{1, m}=F_{2, m}, F_{2, m-1}, \ldots, F_{2,0}=F_{3,0} \\
F_{3,1}, \ldots, F_{3, m}, \ldots, F_{n, 0}
\end{gathered}
$$

after some deletions and trivial insertions, is a sequence of Jónsson operations for $\boldsymbol{V}(\mathbf{A})$. 


\section{EQUIVALENT CONDITIONS FOR CONGRUENCE DISTRIBUTIVITY}

The principal result of this section will be a characterisation of the finite upper bounded ordered sets whose corresponding order-primal variety is congruencedistributive, in terms of the existence of a finite system of monotone binary operations satisfying certain inclusion relations and equations. But first, we note that congruence modularity and congruence-distributivity are equivalent for the order-primal varieties corresponding to finite ordered sets. Let $\mathbf{P}$ be a finite ordered set and assume that $V(A(P))$ is congruence-modular. In the remarks introducing Theorem 1.3 we observed that $\mathbf{A}(\mathbf{P})$ has no proper subalgebras and has at most three congruences. It is thus easy to verify that when $\alpha<\beta$ are congruences of $\mathbf{A}(\mathbf{P})$ then $\beta$ fails to be $A$ belian over $\alpha$. Hence the commutator on congruences of $\mathbf{A}(\mathbf{P})$ satisfies $[\theta, \psi]=\theta \cap \psi$. The algebra $\mathbf{A}(\mathbf{P})$ is hereditarily neutral, in the terminology of Hagemann and Herrmann [10]. According to their Corollary 4.2, a finite hereditarily neutral algebra in a congruence modular variety generates itself a congruence-distributive variety. (For a detailed proof of this fact, see [9, Exercise 2, p.89 and p.199].) Hence we have the following result.

TheOREM 2.1. If $\mathbf{A}$ is a finite order-primal algebra then $\mathbf{V}(\mathbf{A})$ is congruencemodular if and only if it is congruence-distributive.

Theorem 2.2. Let $\mathbf{A}$ be monotone with respect to a possibly infinite ordered set $\mathbf{P}$ and suppose that $\mathbf{A}$ has a term operation $u(x, y)$ such that $u(x, y)$ is an upper bound of $x$ and $y$ for all $x, y \in P$. Then $V(\mathbf{A})$ is congruence-modular if and only if it is congruence-distributive.

Proof: Assume that $\boldsymbol{V ( A )}$ is congruence-modular, but not congruencedistributive. Then there is an algebra $B \in V(A)$ and a congruence $\theta$ on $B$ such that $\psi=[\theta, \theta]<\theta$. In fact, there is such an algebra $\mathbf{B}$ which is a subalgebra of $\mathbf{A}^{X}$ for some set $X$. We recall that there is a term $p(x, y, z)$ (the difference term; see [ 8 , Theorem 5.5]) satisfying $p(x, x, y) \approx y$ and such that whenever $(x, y) \in \theta$ then $p(x, y, y) \equiv x(\bmod [\theta, \theta])$. Choose any $(a, b) \in \theta-\psi$. Now $B$ is ordered as a subset of $\mathbf{P}^{X}$, and the term operations of $\mathrm{B}$ are monotone for this ordering. We have $b=p(u(a, b), u(a, b), b) \geqslant p(a, b, b)=u$ and $u \equiv a(\bmod \psi)$. Then $b=p(u, u, b) \leqslant p(u, b, b) \leqslant p(b, b, b)=b$ and so $b=p(u, b, b)$. But since $(u, b) \in \theta$ it follows that $p(u, b, b) \equiv u \equiv a(\bmod \psi)$. This contradicts our choice of $(a, b) \notin \psi$, and proves the theorem.

Davey [2] proved the equivalence of congruence-modularity and congruencedistributivity for varieties generated by $\mathbf{P}$-monotone algebras under various assumptions not covered in our two preceding theorems; for example, under the assumption that $\mathbf{P}$ is upward and downward directed. 
Suppose that $\mathbf{P}$ is an ordered set (not necessarily finite) with 1 , and that $\mathbf{M}(\mathbf{P})$ contains operations $d_{0}, \ldots, d_{n}$ satisfying all of Jónsson's equations except $d_{i}(x, y, x) \approx$ $x$, and satisfying $d_{i}(x, 1, x) \approx x$. We observe that $M(P)$ must contain operations $b_{0}(x, y), \ldots, b_{m}(x, y)$ satisfying

$$
\begin{array}{r}
x=b_{0}(x, y)=b_{i}(x, x)=b_{m}(y, x)(\text { for } 0 \leqslant i \leqslant m) \\
b_{2 i}(x, y) \leqslant b_{2 i+1}(x, y)(\text { for } 0 \leqslant i \leqslant(m-1) / 2) \\
b_{2 i+1}(x, y) \geqslant b_{2 i+2}(x, y)(\text { for } 0 \leqslant i \leqslant(m-2) / 2) .
\end{array}
$$

In fact, we can take $m=2 n$ and

$$
\begin{aligned}
b_{0}(x, y) & =x=d_{1}(x, x, y), b_{1}(x, y)=d_{1}(x, 1, y), \\
b_{2}(x, y)=d_{1}(x, y, y) & =d_{2}(x, y, y), b_{3}(x, y)=d_{2}(x, 1, y), \\
b_{4}(x, y) & =d_{2}(x, x, y)=d_{3}(x, x, y)
\end{aligned}
$$

and so on. Then the inclusions and equations in (11) are obviously satisfied.

For any finite ordered set $\mathbf{P}$, we shall now prove that the existence of a system of binary monotone operations satisfying (11) is a sufficient condition for the existence of monotone Jónsson operations. We shall observe in Theorem 2.4 , that if $\mathbf{P}$ has 0 and 1 then the condition is equivalent to the property that any two elements $x$ and $y$ are connected in the convex hull of $\{x, y\}$, holding not only in $\mathbf{P}$, but in every subdirect power of $\mathbf{A}(\mathbf{P})$ under the ordering inherited from the Cartesian product.

Theorem 2.3. A finite ordered set $\mathbf{P}$ that admits a system of monotone operations satisfying (11) admits monotone Jónsson operations.

Proof: Assume that $b_{0}(x, y), \ldots, b_{m}(x, y) \in \mathrm{M}(\mathbf{P})$ satisfy (11). We note that it cannot be possible to obtain Jónsson operations as compositions of the $b_{i}$, for the two-element semilattice has term operations satisfying (11), but does not have Jónsson term operations. Our proof will be a rather complicated recursive construction of the Jónsson operations, using many case-by-case definitions.

Our first step will be to modify the $b_{i}(x, y)$ to obtain a new system satisfying (11) that possesses some nice additional properties. (This part of our argument does not seem capable of being generalised to the case of bounded, possibly infinite, ordered sets of finite height.) First, by iterating all operations in the first variable, we can obtain a system satisfying (11) and also

$$
b_{i}\left(b_{i}(x, y), y\right)=b_{i}(x, y) \text { for all } i
$$

In more detail, define $b_{i}^{(0)}(x, y)=b_{i}(x, y)$ and, inductively, $b_{i}^{(j+1)}(x, y)=$ $b_{i}\left(b_{i}^{(j)}(x, y), y\right)$. Choose an $n$ such that $b_{i}^{(n)}\left(b_{i}^{(n)}(x, y), y\right)=b_{i}^{(n)}(x, y)$ for all $i \leqslant m$ 
and all $x, y \in P$. Then replace the $b_{i}(x, y)$ by $b_{i}^{(n)}(x, y)$. (For more details on how this works, see Lemma 4.4 in [11].)

Note that $x \geqslant y$ implies $b_{0}(x, y)=b_{1}(x, y)$ (since $x=b_{0}(x, y) \leqslant b_{1}(x, y) \leqslant$ $b_{1}(x, x)=x$ if $x \geqslant y$ ). Our next goal is find a system of monotone operations for which (11) and (12) hold, and also

$b_{2 i}(x, y) \geqslant y$ implies $b_{2 i}(x, y)=b_{2 i+1}(x, y)$ (for all $\left.i \leqslant(m-1) / 2\right) ; \quad$ and

$b_{2 i+1}(x, y) \leqslant y$ implies $b_{2 i+1}(x, y)=b_{2 i+2}(x, y)$ (for all $i \leqslant(m-2) / 2$ ).

To move toward the satisfaction of (13), replace $b_{i}(x, y)$ by $b_{i}\left(b_{1}(x, y), y\right)$, for $1 \leqslant i \leqslant m$. This replacement does not change $b_{1}(x, y)$ (in view of (12)), preserves (11), destroys (12), and ensures that

$$
b_{i}\left(b_{1}(x, y), y\right)=b_{i}(x, y) \text { for all } i \geqslant 1
$$

Now iterate all functions in the first variable to achieve (12) again. The equations displayed immediately above are still true. Next, replace $b_{i}(x, y)$ by $b_{i}\left(b_{2}(x, y), y\right)$ for all $i \geqslant 2$, and then iterate to regain (12), as well as

$$
b_{i}\left(b_{j}(x, y), y\right)=b_{i}(x, y) \text { when } j \in\{0,1,2\} \text { and } i \geqslant j
$$

Continuing in this fashion we eventually produce $b_{0}, \ldots, b_{m}$ satisfying (12) and also

$$
b_{i}\left(b_{j}(x, y), y\right)=b_{i}(x, y) \text { whenever } i \geqslant j \text {. }
$$

We claim that (11) and (14) imply (13). To see it, suppose first that $i<m$ is odd and $b_{i}(x, y) \leqslant y$. then

$$
b_{i+1}(x, y)=b_{i+1}\left(b_{i}(x, y), y\right) \geqslant b_{i+1}\left(b_{i}(x, y), b_{i}(x, y)\right)=b_{i}(x, y)
$$

and so $b_{i+1}(x, y)=b_{i}(x, y)$ since the reverse inclusion is part of (11). The other half of (13) follows by an analogous calculation.

The operations $b_{i}$ are now as nice as we need them to be. What we require is that they satisfy (11) and (13). The remainder of the proof is valid for bounded ordered sets of finite height. We need to partition $P$ into "levels" in two ways. Let $D_{k}$ be the set of all $x \in P$ such that every chain with top element $x$ has at most $k+1$ elements. Thus there is an integer $m_{0}$ such that

$$
D_{0} \subseteq D_{1} \subseteq D_{2} \subseteq \cdots \subseteq D_{m_{0}}=P
$$

and the sets $D_{0}, \ldots D_{m_{0}}$ are distinct. Similarly, define $U_{k}$ to be the set of all $x \in P$ such that every chain with $x$ as bottom element has at most $k+1$ elements. Thus 
$U_{m_{1}-1}<U_{m_{1}}=P$. Now if $u<v$ in $P$, then $u \in U_{i+1}$ implies $v \in U_{i}$; and $v \in D_{j+1}$ implies $u \in D_{j}$.

The Jónsson operations $d(x, y, z)$ that we are about to construct are defined by cases in such a way that for every $(a, b, c) \in P^{3}$ there is an $i \leqslant m$ with $d(a, b, c)=$ $b_{i}(a, c)$. Thus the equation $d(x, y, x)=x$ is guaranteed to be true. It will need a bit of cleverness, however, to ensure that the operations are monotone, and satisfy all of Jónsson's equations. For each $i \leqslant m$, we shall have $b_{i}(x, z)=d_{j}(x, y, z)$ for a certain $j$ and for all $x, y, z$. The trick will be to figure out how to connect $b_{i}(x, z)$ to $b_{i+1}(x, z)$ by a string of monotone operations satisfying the (alternating) Jónsson equations. For $B \subseteq P$ we define $B^{\star}$ to be the set of all upper bounds of $B$, and $B_{\star}$ to be the set of all lower bounds of $B$.

We begin by connecting $b_{0}(x, z)$ and $b_{1}(x, z)$. Let $d_{0}(x, y, z)=x=b_{0}(x, z)$. Then define $d_{1}(x, y, z)$ to be $x$ unless it is the case that $\{x, y\}^{\star} \cup\{x, z\}^{\star} \subseteq U_{0}$ and in this case let $d_{1}(x, y, z)=b_{1}(x, z)$. It is obvious that $d_{1}$ is monotone (the fact that $b_{1}(x, y) \geqslant x$ is used here). Also, we have that $d_{1}(x, x, y)=x=d_{0}(x, x, y)$ (since if $\{x, x\}^{\star} \subseteq U_{0}$ then $x$ is maximal and $x \leqslant b_{1}(x, y)$ implies $x=b_{1}(x, y)$ ), and $d_{1}(x, y, x)=x$ (for the reason mentioned above). Next, let $d_{2}(x, y, z)=d_{1}(x, z, z)$.

Now it gets a little more tricky. We define $d_{3}(x, y, z)=x$ unless either $\{x, z\}^{\star} \subseteq U_{0}$ or else $\{x, y\}^{\star} \cup\{x, z\}^{\star} \subseteq U_{1}$, and put $d_{3}(x, y, z)=b_{1}(x, z)$ when this condition holds. The proof that $d_{2}(a, a, c)=d_{3}(a, a, c)$ goes as follows. If $\{a, a\}^{\star} \cup\{a, c\}^{\star} \subseteq U_{1}$, this just means that $a \in U_{1}$; then if $\{a, c\}^{\star} \subseteq U_{0}$ fails it follows, since $a \in U_{1}$, that $c \leqslant a$; but in this case $b_{1}(a, c)=a$ by $(13)$ and so $d_{3}(a, a, c)=d_{2}(a, a, c)$. Next, we define $d_{4}(x, y, z)=d_{3}(x, z, z)$.

We are now ready to define the remaining operations in the string from $b_{0}$ to $b_{1}$. For each $2 \leqslant i \leqslant m_{1}$ we define $d_{2 i+1}(x, y, z)$ to be $x$ unless $\{x, z\}^{\star} \subseteq U_{i-1}$ or $\{x, y\}^{\star} \cup\{x, z\}^{\star} \subseteq U_{i}$, in which case $d_{2 i+1}(x, y, z)=b_{1}(x, z)$. We define $d_{2 i+2}(x, y, z)=$ $d_{2 i+1}(x, z, z)$. Now we have $d_{2 m_{1}+1}(x, y, z)=b_{1}(x, z)$ (for all arguments). The proof that $d_{0}, d_{1}, \ldots, d_{2 m_{1}+1}$ satisfy the relevant Jónsson equations follows the same pattern as the proof for $d_{0}, d_{1}, d_{2}, d_{3}, d_{4}$ which has already been given. Let us redenote these operations by $d_{i}^{(0)}$ in recognition of the fact that they get us from $b_{0}$ to $b_{1}$.

To stretch a string of Jónsson operations from $b_{1}$ to $b_{2}$, we need to start at the bottom and work up through the "levels" $D_{i+1}-D_{i}$, since $b_{1} \geqslant b_{2}$. Each of the operations $d_{i}$ in our string will be a composite of $b_{1}$ and $b_{2}$, so that $d_{i}(x, y, z)$ equals $b_{2}(x, z)$ on a certain order-ideal of $P^{3}$, and equals $b_{1}(x, z)$ on the complementary order-filter. Of course, we define $d_{0}(x, y, z)=b_{1}(x, z)$. We define $d_{1}(x, y, z)$ to be $b_{1}(x, z)$ unless $\left\{b_{1}(x, z), z\right\}_{\star} \cup\left\{b_{1}(x, z), b_{1}(y, z)\right\}_{\star} \subseteq D_{0}$, and then to be $b_{2}(x, z)$. We define $d_{2}(x, y, z)=d_{1}(x, z, z)$. Then for $1 \leqslant i \leqslant m_{0}$ we define $d_{2 i+1}(x, y, z)$ to be 
$b_{1}(x, z)$ unless

$$
\begin{gathered}
\left\{b_{1}(x, z), z\right\}_{\star} \subseteq D_{i-1} \text { or } \\
\left\{b_{1}(x, z), z\right\}_{\star} \cup\left\{b_{1}(x, z), b_{1}(y, z)\right\}_{\star} \subseteq D_{i},
\end{gathered}
$$

in which event $d_{2 i+1}(x, y, z)=b_{2}(x, z)$. We define $d_{2 i+2}(x, y, z)=d_{2 i+1}(x, z, z)$.

Now we have $d_{2 m_{0}+1}(x, y, z)=b_{2}(x, z)$. The argument to show that the relevant Jónsson equations are satisfied relies on one part of (13), namely the fact that when $b_{1}(x, z) \leqslant z$ then $b_{1}(x, z)=b_{2}(x, z)$. Let us redenote the operations we have just constructed, as $d_{i}^{(1)}, 0 \leqslant i \leqslant 2 m_{0}+1$.

The remainder of the construction is visible in what we have already done. The final system of Jónsson operations takes the form (where $r=2 m_{1}+1$ and $s=2 m_{0}+1$ )

$$
\begin{array}{r}
d_{0}^{(0)}, \ldots, d_{r}^{(0)}=d_{0}^{(1)}, \ldots, d_{z}^{(1)}=d_{0}^{(2)}, \ldots \\
d_{0}^{(m-1)}, \ldots, d_{t}^{(m-1)}(t \in\{r, s\})
\end{array}
$$

in which $d_{0}^{(i)}(x, y, z)=b_{i}(x, z)$.

We remark that if $\mathbf{Q}$ is a subalgebra of $\mathbf{A}(\mathbf{P})^{\boldsymbol{X}}$ where $\mathbf{P}$ is an ordered set, then $\mathbf{Q}$ is naturally an ordered set, under the product ordering inherited from $\mathbf{P}^{X}$. If $\{x, y\} \subseteq S \subseteq Q$ where $\mathbf{Q}$ is an ordered set, we say that $x$ and $y$ are connected in $S$ if and only if there exist elements $z_{0}, \ldots, z_{n}$ in $S$ (for some $n$ ) such that $z_{0}=x \leqslant z_{1} \geqslant$ $z_{2} \leqslant \cdots \geqslant z_{n}=y$. Recall that $S^{\star}$ and $S_{\star}$ are, respectively, the set of all upper bounds of $S$ and the set of all lower bounds of $S$. By the convex hull of $S$, we shall mean the set $\left(S_{\star}\right)^{\star} \cap\left(S^{\star}\right)_{\star}$.

Theorem 2.4. For any finite ordered set $\mathbf{P}$ with 1 , conditions (a) $-(d)$ are equivalent. If $\mathbf{P}$ has 0 and 1 then all the conditions are equivalent to (e).

(a) The variety generated by $\mathbf{A}(\mathbf{P})$ is congruence-modular.

(b) The variety generated by $\mathbf{A}(\mathbf{P})$ is congruence-distributive.

(c) The variety generated by $\mathbf{A}(\mathbf{P})$ is residually small.

(d) $\mathbf{P}$ supports monotone operations satisfying (11).

(e) If $\mathbf{Q}$ is a subalgebra of $(\mathbf{A}(\mathbf{P}))^{n}$ for some positive integer $n$ then in the ordered set $Q$, any two elements $x$ and $y$ are connected in the convex hull of $\{x, y\}$.

Proof: The equivalence of (a) and (b) is contained in both Theorem 2.1 and Theorem 2.2. That (c) implies (d) is a consequence of the dual of Lemma 1.4 and our remarks preceeding Theorem 2.3. The implication (d) implies (b) is contained in Theorem 2.3, and (b) implies (c) is a consequence of Jonsson's classical work. The proof of $(\mathrm{d}) \Rightarrow$ (e) is trivial; since $b_{i}^{\mathbf{Q}}$ are monotone and idempotent, the elements $b_{i}^{\mathbf{Q}}(x, y)$ must all belong to the convex hull of $\{x, y\}$. 
Now assuming that $P$ has both 0 and 1 , we prove that that $(e) \Rightarrow(d)$. Let $Q$ be the subalgebra of $(\mathbf{A}(\mathbf{P}))^{P^{2}}$ whose universe is the set of binary members of $\mathrm{M}(\mathbf{P})$. Let $f_{0}(x, y)=x$ and $f_{1}(x, y)=y$ and let $D=\left\{f_{0}, f_{1}\right\}_{\star} \subseteq Q$ and let $U=\left\{f_{0}, f_{1}\right\}^{\star}$. Applying (e) to this situation, there exist elements $b_{0}=f_{0}, b_{1}, \ldots, b_{m}=f_{1}$ in $Q$ such that $b_{0} \leqslant b_{1} \geqslant b_{2} \leqslant b_{3} \ldots \geqslant b_{m}$ and every element $b_{i}$ is bounded above by $U$ and below by $D$. Now the $b_{i}$ belong to $M(P)$ and it is nearly obvious that they satisfy (11). The only point that may be non-obvious is the claim that $b_{i}$ are idempotent, that is, $b_{i}(x, x)=x$. To see that they are, let $a \in P$, and construct an element of $Q$ as follows. Define $f(x, y)$ to be 1 unless $x \leqslant a$ and $y \leqslant a$, and in that case put $f(x, y)=a$. Clearly, $f \in Q$ and $f(x, y) \geqslant x$ for all $x$ and $y$; that is, $f \geqslant f_{0}$. Likewise, $f \geqslant f_{1}$; and so $f \in U$ and consequently $f \geqslant b_{i}$. Then it follows that $b_{i}(a, a) \leqslant f(a, a)=a$. A parallel argument, employing the zero element, proves that $b_{i}(a, a) \geqslant a$.

Remarks. Our results in Section 1 and Section 2 are not as strong as one would like and, unfortunately, we have no examples demonstrating that stronger results are not valid. So we are left with several open questions. Are Theorem 1.1 and Corollary 1.2 true without any boundedness requirement? Is Theorem 2.1 true for all (finite and infinite) order-primal algebras? Davey [2] contains a wealth of results and examples bearing on the second problem.

In regard to the possibility of strengthening Theorem 2.3, we remark that, if we have monotone Jónsson operations $d_{0}(x, y, z), \ldots, d_{n}(x, y, z)$ for the ordered set $\mathbf{P}$, then $b_{0}(x, y)=x, b_{1}(x, y)=d_{1}(x, y, y), b_{2}(x, y)=d_{2}(x, x, y), b_{3}(x, y)=d_{3}(x, y, y), \ldots$, $b_{n}(x, y)=y$ satisfy a weakening of $(11)$, namely

$$
\begin{gathered}
x=b_{0}(x, y)=b_{i}(x, x)=b_{n}(y, x) \quad(\text { for } 0 \leqslant i \leqslant n) ; \text { and } \\
\text { for } i<n \text {, if }\{x, y\}^{\star} \neq \emptyset \text { then the convex hull of }\{x, y\} \\
\text { intersects }\left\{b_{i}(x, y), b_{i+1}(x, y)\right\}^{\star} ; \text { and dually. }
\end{gathered}
$$

For finite $\mathbf{P}$, does the existence of monotone operations satisfying (11') imply the existence of monotone Jónsson operations?

\section{EXAMPLES AND COMMENTARY}

An $n$-ary near unanimity function, or $n-n u f$, on $X$ is an operation $f\left(x_{1}, \ldots, x_{n}\right)$ of $n$ variables over $X$ obeying the equations

$$
f(y, x, \ldots, x) \approx f(x, y, x, \ldots, x) \approx \ldots \approx f(x, x, \ldots, x, y) \approx x
$$

Jónsson operations on $X$ can immediately be derived from an $n$-nuf when $n \geqslant 3$, by defining $d_{2 i-1}(x, y, z)=f(z, \ldots, z, y, x, x, \ldots, x)$ with the unique $y$ occuring at the 
place of $x_{i+1}$, and defining $d_{2 i}(x, y, z)=d_{2 i-1}(x, z, z)$, for $1 \leqslant i<n-1$. (With this definition, $d_{1}(x, x, y)=x$ and $d_{2(n-2)}(x, y, z)=z$.) Thus an ordered set $P$ that admits a nuf (that is, a monotone $n$-nuf for some $n \geqslant 3$ ) admits Jónsson operations. We do not know if the converse is true, even if we assume that $\mathbf{P}$ is finite. The problem of characterising the finite ordered sets that admit a nuf has received a lot of attention in the literature of the past five years; we shall review a few of the known results near the end of this section.

For any finite ordered set $P$, it is of interest to know whether the clone $M(P)$ is finitely generated. It follows from classical results of Baker and Pixley $[1]$ that $M(P)$ is finitely generated if it contains an $n$-nuf for some $n \geqslant 3$. The ordered set $T$ pictured in Figure 1 has played an interesting rôle in the investigation of algebraic properties of ordered sets; several seemingly plausible conjectures were first disproved with a deeper study of the monotone operations on $T$. Tardos [22] proved that $M(T)$ is not finitely generated, thus also showing that $T$ admits no nuf; while Lau [14] proved that $M(P)$ is finitely generated whenever $\mathbf{P}$ is a bounded ordered set of fewer than eight elements. Demetrovics, Hannák and Rónyai $[\mathbf{5}]$ proved that $\boldsymbol{V}(\mathbf{A}(\mathbf{T}))$ is not congruence-modular. This fact follows easily from our Theorem 2.4; the two elements at middle height in $T$ are not connected in their convex hull.

The ordered sets of Figure 1 differ greatly in the quality of idempotent monotone operations they admit. $\mathbf{P}_{2}$ has a 5-nuf but no 4-nuf (see below). As we remarked,
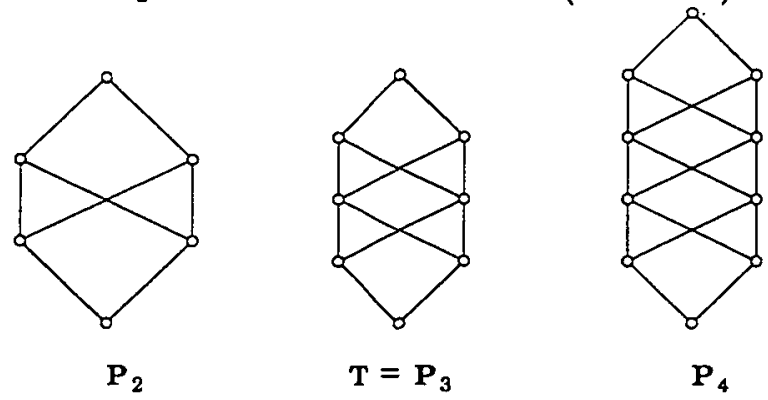

$\mathbf{T}=\mathbf{P}_{3}$

$\mathbf{P}_{4}$

Figure 1

$P_{3}(=\mathbf{T})$ does not admit Jónsson operations; however, it does admit monotone ternary operations obeying the equations

$$
\begin{gathered}
x=d_{1}(x, x, y), d_{1}(x, y, y)=d_{2}(x, y, y), d_{1}(x, y, x)=d_{2}(x, y, x) \\
d_{2}(x, x, y)=d_{3}(x, x, y), d_{3}(x, y, x)=d_{3}(y, x, x)=x .
\end{gathered}
$$

This implies that the variety generated by $\mathbf{A}\left(\mathbf{P}_{3}\right)$ is congruence semi-distributive (see [11, Theorem 9.11]). To define $d_{1}, d_{2}, d_{3}$, we use $D, M$ and $U$ to denote the subsets of $P_{3}$ consisting of the bottom three elements, the middle two elements, and the top 
three elements, respectively. We define $d_{1}(x, y, z)$ to be $x \vee y$ if $\{x, y\} \cap U \neq \emptyset$; to be $x \wedge y$ if $\{x, y\}$ is disjoint from $U$ but intersects $D$; and to be $x$ otherwise. (Here, $x \vee y$ denotes the join, or least upper bound, of $x$ and $y$, and $x \wedge y$ denotes their meet. These joins and meets obviously exist under the stated conditions.) Then we define $d_{2}(x, y, z)$ to be $x \vee y \vee z$ if $\{x, y, z\}$ intersects $U$; to be $x \wedge y \wedge z$ if $\{x, y, z\}$ intersects $D$ and is disjoint from $U$; and otherwise to be $x$ if $x \neq y$ and $z$ if $x=y$. We define $d_{3}(x, y, z)$ to be $(x \vee z) \wedge(y \vee z)$ if $z \in U$; to be $x \wedge y$ if $x$ and $y$ are comparable and belong to $U$ while $z$ does not belong to $U$; to be $(x \wedge z) \vee(y \wedge z)$ if $\{x, y, z\} \cap U=\emptyset$ and $z \in D$; to be $x \vee y$ if $x$ and $y$ are comparable and belong to $D$ while $z \in M$; and to be $z$ otherwise. The reader can verify that these operations satisfy the equations (15) and are monotone.

If $f\left(x_{1}, \ldots, x_{n}\right)$ is an idempotent operation in $\mathrm{M}\left(\mathbf{P}_{4}\right)$ then the set of four midlevel elements of $\mathbf{P}_{4}$, being its own convex hull, is closed under $f$. It follows by a result from $[\boldsymbol{B}]$ mentioned below that when its variables are restricted to range over these four elements, $f\left(x_{1}, \ldots, x_{n}\right)$ becomes a projection; that is, $f\left(x_{1}, \ldots, x_{n}\right)=x_{i}$ for some fixed $i$. Thus $\mathrm{P}_{4}$ cannot satisfy the Maltsev condition represented by the equations (15), and in fact cannot satisfy any Maltsev condition involving idempotent operations except those (trivial) Maltsev conditions that are satisfied by the variety of sets with no operations.

Of course, if $P$ is lattice-ordered, then it has a 3-nuf. In [4] one finds the observation that if $\mathbf{P}$ is bounded but not lattice-ordered then it admits no 3 -nuf, and admits no 4-nuf if it is of finite height. For if $m(x, y, z)$ were a monotone 3-nuf for $\mathbf{P}$ then $m(a, 1, b)$ would have to be the least upper bound of $a$ and $b$; and if $f(x, y, z, u)$ were a monotone 4-nuf for $\mathbf{P}$ and if $c, d$ are two distinct minimal members of $\{a, b\}^{\star}$ then $f(a, b, c, d)=u$ would satisfy $u \in\{a, b\}^{\star} \cap\{c, d\}_{\star}$. In [4] one finds also the result that if $P$ is an ordered set obtained by removing from a lattice $L$ a subset $H$ that is convex in the sense that $x<y<z,\{x, z\} \subseteq H$ imply $y \in H$, and if $\mathrm{L}$ has no chain of $k+1$-elements, then $\mathbf{P}$ has a $2 k+1$-nuf. The existence of a 5 -nuf on $\mathbf{P}_{2}$ is a consequence of this result; it has been generalised in [19] to the result that any finite bounded ordered set of height $\leqslant 3$ has a 5 -nuf.

The ordered sets known as fences possess 3 -nuf's. Two fences, $F_{2}$ and $F_{5}$, are pictured in Figure 2. There is precisely one monotone 3-nuf on the fence $\mathbf{F}_{n}$, and it can be defined with the aid of the visible notion of between-ness. For $x, y, z$ elements of $F_{n}$, say that $y$ is between $x$ and $z$ if every path from $x$ to $z$ in the covering graph of the fence includes $y$. Then define $m(a, b, c)$ to be the unique $x \in\{a, b, c\}$ which is between the other two elements. In [19] it is proved that a finite ordered set admits a 3 -nuf if and only if it is a retract of a product of finite fences. There is no known characterisation of finite ordered sets that admit an $n$-nuf if $n$ is a fixed integer greater 


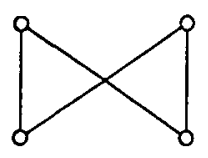

$\mathrm{C}_{2}$

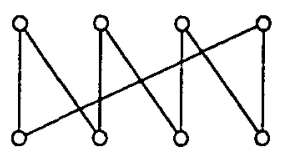

$\mathrm{C}_{4}$

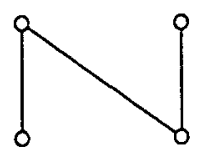

$\mathbf{F}_{2}$

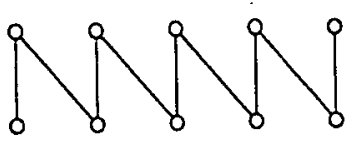

$\mathbf{F}_{5}$

Figure 2

than 3, or of finite ordered sets that admit an $n$-nuf for some $n \geqslant 3$.

The ordered sets $C_{2}$ and $C_{4}$ pictured in Figure 2 are called crowns. All the crowns $\mathbf{C}_{n}(n \geqslant 2)$ have some unusual properties. Demetrovics and Rónyai [6] have shown that the clone of $\mathbf{C}_{n}$ is finitely generated; moreover, this clone is contained in the Slupecki clone consisting of all operations on the given set that either have proper range, or depend on at most one variable. Thus a crown admits no non-trivial idempotent operation. This is the basis of our earlier claim about $\mathbf{P}_{4}$ (Figure 1 ).

The ordinal sum $1+2+C_{2}+2+1$, where 1 and 2 denote one and two-element discretely ordered sets, is $\mathbf{P}_{4}$. The ordinal sum $1+2+\mathbf{F}_{n}+2+1$ is a bounded ordered set we denote by $\mathbf{L} \mathbf{F}_{n}$, and call a locked fence. Two locked fences are pictured in Figure 3. For a long time, we suspected that locked fences might supply examples of ordered sets that admit Jónsson operations but no nuf's. However, we eventually discovered that they do admit nuf's, although it does not seem to be an easy matter to construct one. We leave this task as an exercise for the reader.

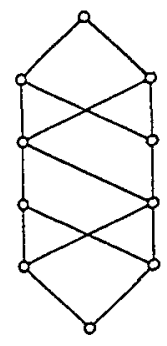

$\mathbf{L F}_{2}$

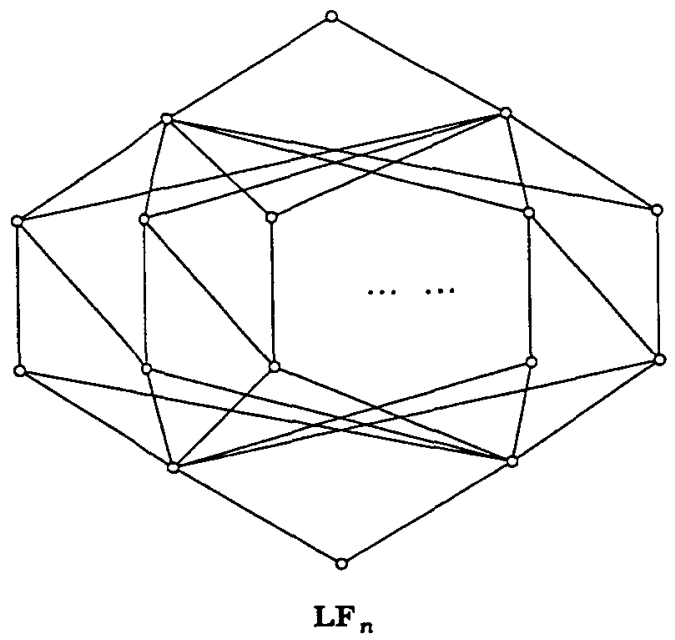

Figure 3

\section{REFERENCES}

[1] K. Baker and A. Pixley, 'Polynomial interpolation and the Chinese Remainder Theorem for algebraic systems', Math. $Z .43$ (1975), 165-174.

[2] B. Davey, 'Monotone clones and congruence modularity', (preprint). 
[3] B. Davey R.W. Quackenbush and D. Schweigert, 'Monotone clones and the varieties they determine', (preprint).

[4] J. Demetrovics, L. Hannák and L. Rónyai, 'Near unanimity functions of partial orderings', in Proceedings of the XIVth International Symposium on Multiple Valued Logic, pp. 52-56 (Manitoba, 1984).

[5] J. Demetrovics, L. Hannák and L. Rónyai, 'On algebraic properties of monotone clones', Order 3 (1986), 219-225.

[6] J. Demetrovics, L. Rónyai, 'Algebraic properties of crowns and fences', (preprint of the Computer and Automation Institute, Hungarian Academy of Sciences, Budapest).

[7] K. Denecke, Preprimal A lgebras (Math. Forschung Bd. 11 Akademie Verlag, Berlin, 1982).

[8] D. Duffus and I. Rival, 'A structure theory for ordered sets', Discrete Math. 35 (1981), 53-118.

[9] R. Freese and R. McKenzie, Commutator theory for congruence modular varieties (London Math. Soc. Lecture Note No. 125, 1987).

[10] J. Hagemann and C. Herrmann, 'A concrete ideal multiplication for algebraic systems and its relation to congruence distributivity', Arch. Math. (Basel) (1979), 234-245.

[11] D. Hobby and R. McKenzie, The Structure of Finite Algebras (AMS Contemporary Mathematics Series, 1988).

[12] B. Jónsson, 'Algebras whose congruence lattices are distributive', Math. Scand. 21 (1967), 110-121.

[13] A. Knoebel, The equational classes generated by single functionally precomplete algebras (Amer. Math. Soc. Memoir No. 57, 1985).

[14] D. Lau, 'Bestimmung der Ordnung maximaler Klassen von Functionen der $k$-wertigen Logik', Z. Math. Logik Grundlag. Math. 24 (1978), 79-96.

[15] V.V. Martynjuk, 'Investigation of classes of functions in many-valued logics', (Russian), Problemy Kibernet. 3 (1960), 49-60.

[16] P. Nevermann, 'A note on axiomatizable order varieties', Algebra Universalis 17 (1983), 129-131.

[17] P. Nevermann and I. Rival, 'Holes in ordered sets', Graphs Combin. 1 (1985), 339-350.

[18] P. Nevermann and R. Wille, 'The strong selection property and ordered sets of finite length', Algebra Universalis 18 (1984), 18-28.

[19] R. Quackenbush, I. Rival and I.G. Rosenberg, 'Clones, order varieties, near unanimity functions and holes', (preprint), Order.

[20] I. Rosenberg, 'Über die funktionale Vollständigkeit in den mehrwertigen Logiken', Rozpravy Ceskoslovenské Akad. Ved 80 (1970).

[21] I. Rosenberg, 'Near unanimity orders', (preprint C.R.M. Université de Montréal, 1985).

[22] G. Tardos, 'A not finitely generated maximal clone of monotone operations', Order 3 (1986), 211-218. 
Department of Mathematics

University of California

Berkeley, California 94720

United States of America 